\title{
The Limitations of Anti-arrhythmic Drug Therapy
}

\author{
Katsuro Shimomura, M.D. and Tohru Ohe, M.D.
}

Cases with tachyarrhythmias are presented, in which the limitations of antiarrhythmic drugs are implicated. Discussions are made on when and how the drug in question is considered to be ineffective in a given patient.

$\mathbf{I}^{\mathrm{N}}$

N dealing with the problems of limitations medical treatment by anti-arrhythmic drugs is subject to, main objects of the study are concerned with how to treat the patients with episodes of recurrent tachyarrhythmias by chronic oral administration of these drugs. While treating these patients with a given drug or drugs, indices that can be used as a guideline, consist of 1) doses of drugs, 2) the status of the underlying disease, 3 ) side effects and complications and 4) a clinical

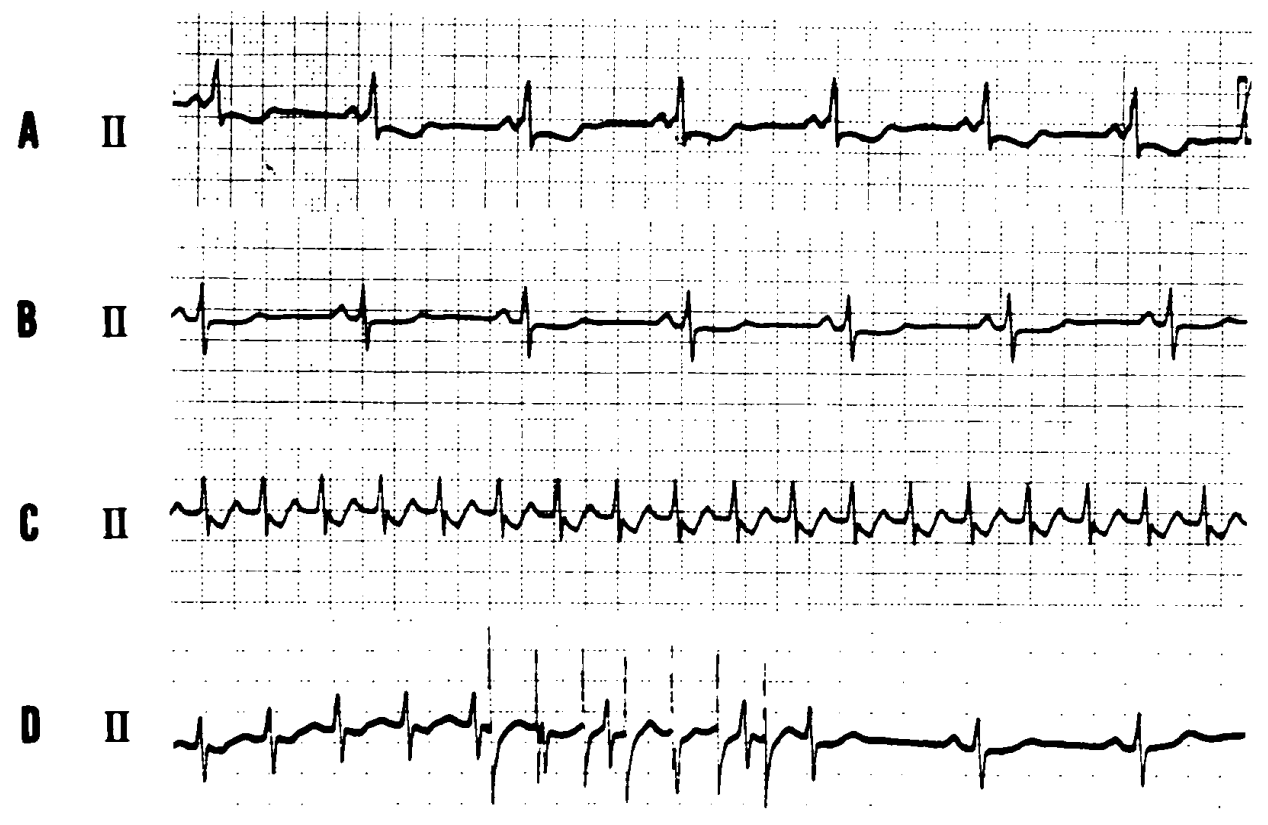

Fig.1. Electrocardiograms in Case 1. A: Sinus rhythm with pre-excitation. B: Sinus rhythm with normal conduction. C: Supraventricular tachycardia. D: Termination of tachycardia by the implanted radio-f requency atrial pacemaker.

\section{Key Words:}

Drug-resistant tachyarrhythmia

Side effect

Anti-arrhythmic drugs

Department of Internal Medicine, National Cardiovascular Center, Suita, Japan

Mailing address: Katsuro Shimomura, M.D., Department of Internal Medicine, National Cardiovascular Center, 5-125 Fuj ishiro-dai, Suita, Osaka 565, Japan 
A $\quad V_{4}$
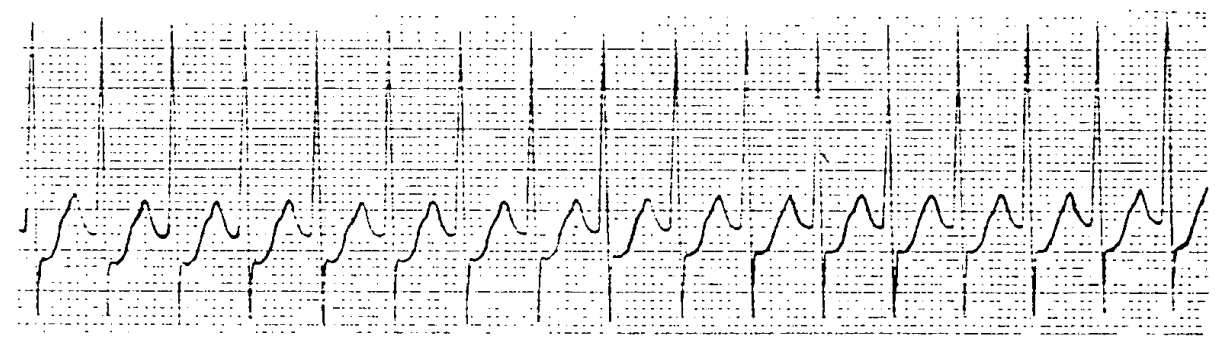

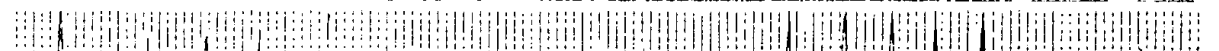

B $\quad V_{4}$
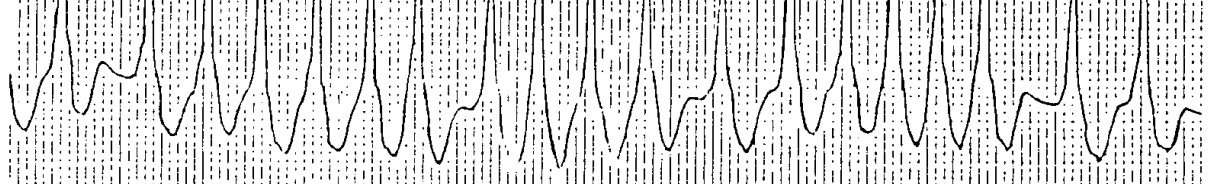

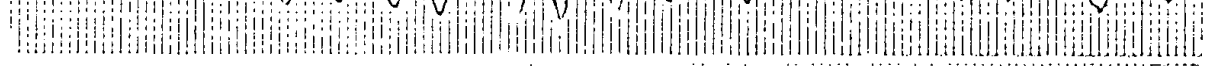

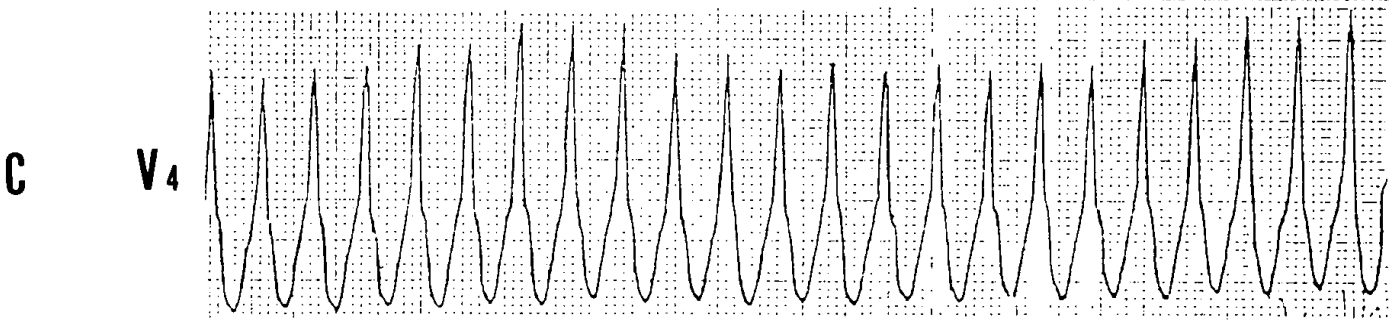

Fig.2. Electrocardiograms in Case 2.

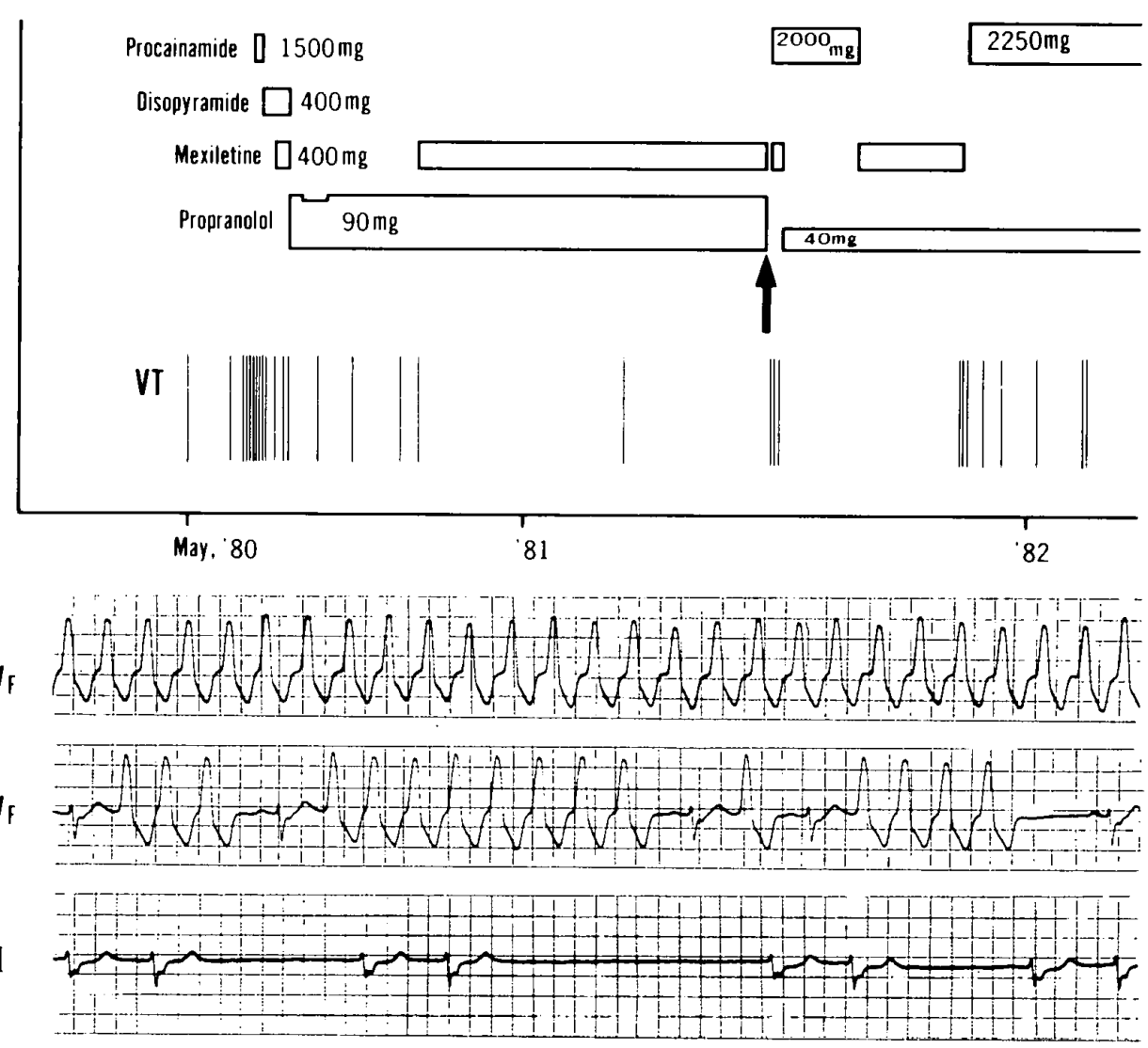

Fig.3. Diagram of treatment and electrocardiograms in Case 3. The arrow shows the time when ECG (C) was recorded. Doses of drugs are expressed as a daily dose.

Japanese Circulation Journal Vol. 47, January 1983 


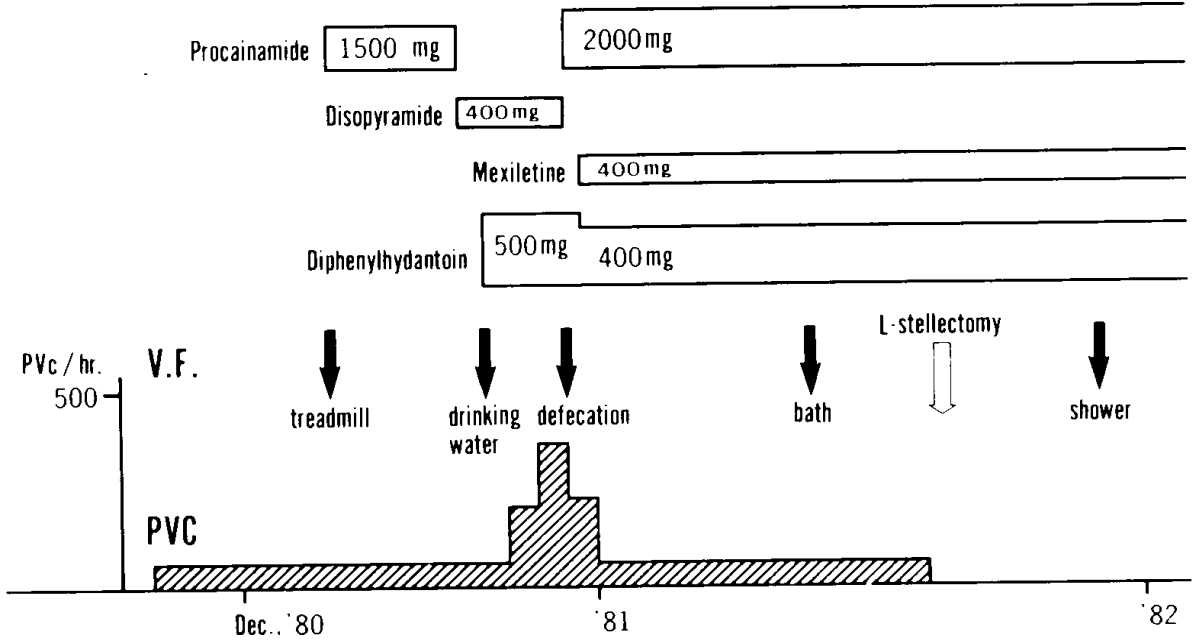

1981

A
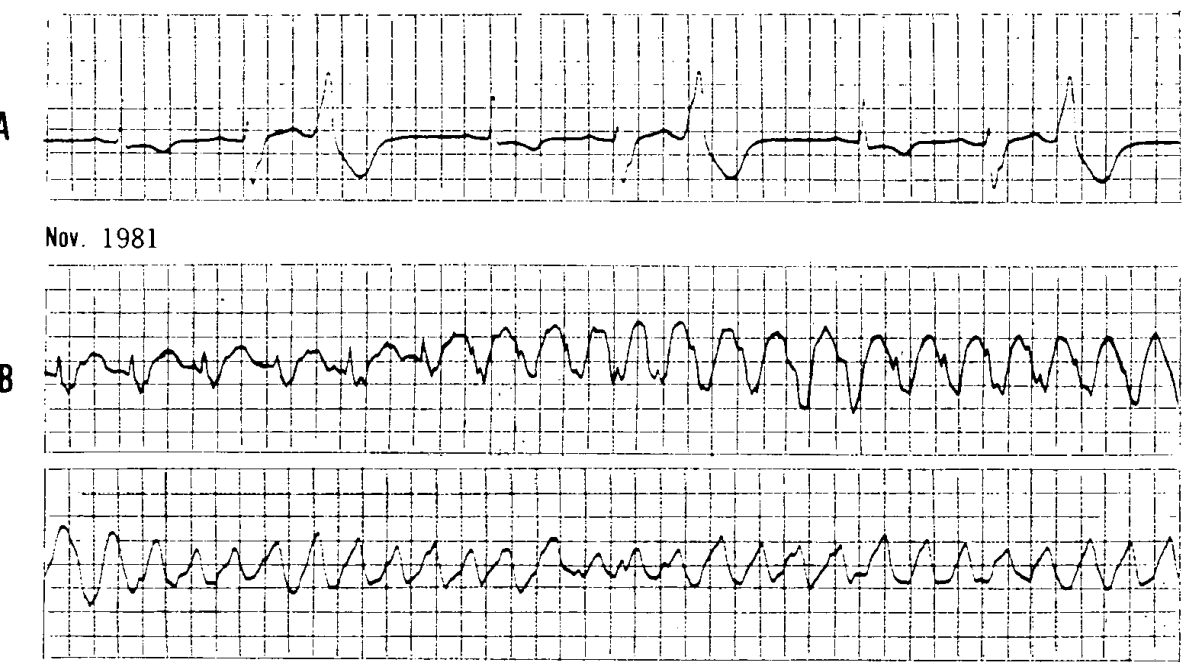

Fig.4. Diagram of treatment and electrocardiograms in Case 4. Doses of drugs are expressed as a daily dose.

impression of being drug-resistant. It is customary with clinical medicine to decide its effect by usual maintenance doses, and to what extent and how long larger doses can be administered is another problem associated with the underlying disease, side effects, safety and so on.

As underlying diseases, cases with ventricular aneurysm or idiopathic cardiomyopathy often develop refractory arrhythmias, while there are some idiopathic cases with tachyarrhythmia which are drug-resistant. There are cases in which anti-arrhythmic drugs cannot be maintained due to side effects or complications, or some additional therapeutic procedures are required for the newly developed symptoms. On the basis of these considerations, it can be deduced that the maintaining dose of the drug is ineffective to a given patient on the following occasions:

1) In a case with paroxysmal supraventricular tachycardia (PSVT), when a) frequency of episodes increases, b) the recurring episodes cannot be controlled and c) episode with severe symptoms cannot be prevented.

2) In a case with WPW syndrome and paroxysmal atrial fibrillation, when an episode of atrial fibrillation with rapid ventricular response cannot be prevented.

3) In a case with ventricular tachycardia, when an episode cannot be prevented.

During the past 3 years we had 23 problematic cases in the treatment of tachyarrhythmias: 6 of 79 cases with PSVT, 9 of 19 cases with WPW syndrome and paroxysmal atrial fibrillation, and 8 of 23 cases with ventricular tachycardia. The problematic cases as referred to here are those with frequent occurrence of episodes, those with severe complications and those with drugresistant tachycardia. Of these cases, surgical 
interventions (aneurysmectomy of the left ventricle or surgical ablation of the accessory pathway) were performed in 10 cases, and an artificial pacemaker was implanted in 6 cases.

\section{CASE PRESENTATION}

\section{Case 1}

A 64-year-old woman with WPW syndrome, who had a history of paroxysmal supraventricular tachycardia of 30 years' duration, visited our hospital because attacks of tachycardia grew more and more frequent with the course of time. Verapamil, quinidine, procainamide, propranolol, disopyramide, digoxin and so on were given singly or in various combinations, without definite effects on each occasion. Since 3 months prior to admission the episodes have become even more frequent, occurring 10 to 20 times per day, which incapacitated her in her daily life. A radio-frequency atrial pacemaker was implanted, and each episode thereafter was terminated by a magnet the patient herself can trigger on initiation of tachycardia, as shown in Fig. 1 .

\section{Case 2}

A 59-year-old man with WPW syndrome had a history of precordial discomfort and palpitation of 10 hours' duration about 20 years ago. $\mathrm{He}$ had been free of complaints thereafter until 14 years prior to admission, when he began to have frequent attacks of palpitation with shock, for which he had been hospitalized. The ECG recordings taken at the time of episodes after admission revealed 3 types of attacks, which were paroxysmal supraventricular tachycardia, pseudoventricular tachycardia due to atrial fibrillation, and atrial flutter of $1: 1$ response with exclusive atrio-ventricular conduction over the accessory pathway (Fig. 2). In case of atrial fibrillation the fastest ventricular rate was $300 / \mathrm{min}$, and in case of atrial flutter the ventricular rate was $250 / \mathrm{min}$. The antegrade effective refractory period of left-sided Kent bundle was $270 \mathrm{msec}$, and $1: 1$ conduction was obtained until atrial pacing rate was increased up to $240 / \mathrm{min}$. The recurrence of episodes persisted in spite of the administration of propranolol, disopyramide, procainamide and so on. Judging from the severity of symptoms and the extremely rapid ventricular rate during episodes, it was considered as perilous to depend on drugs alone for the prevention of palpitation attacks, and surgical ablation of the accessory pathway was performed with success. For some time after surgery paroxysmal atrial fibrillation persisted, but QRS complexes were then of normal conduction and the episodes were well controlled by the administration of digoxin $0.25 \mathrm{mg}$ daily.

\section{Case 3}

A 55-year-old man with no apparent heart disease had frequent occurrence of ventricular tachycardia since a year prior to admission. The ECGs taken during attacks showed two types of tachycardia: one was sustained ventricular tachycardia and the other repetitive type of short runs as shown in Fig. 3 (ECG A and B). Since these episodes tended to occur when the patient was taking a bath or performing a treadmill test, propranolol was given with significant effect. Two months later, he developed ventricular tachycardia, for which mexiletine was administered in combination with propranolol with resultant disappearance of attacks. When he was admitted for the 3rd time, marked bradyarrhythmia was detected (Fig. 3, ECG C). No P waves were discernible, but QRS complexes with CRBBB type appeared in pairs as junctional rhythm, indicating sinus node dysfunction due to the combined effect of these 2 drugs. Mexiletine was then replaced by procainamide, but recurring episodes could not be eliminated entirely at the time of presentation. From the findings of electrophysiological study evidence was obtained to indicate that an ectopic focus of ventricular tachycardia is located at the outflow tract of the right ventricle. Therefore, this case will be a candidate for surgical intervention in the future. In this case also, procainamide was administered in the uppermost dose of $2.25 \mathrm{~g}$ per day. This dose was significantly less than that seen in a recent report, ${ }^{3}$ in which large doses (3.0 to 12 $\mathrm{g}$ /day) were given in order to maintain effective plasma levels despite development of adverse effects like gastrointestinal symptoms, arthralgia, pleuritis or mild immunological abnormalities. Thus, the problem of maintenance dose leads to either of the following: whether limitations border on the appearance of side effects or how far the manifest side effects are to be tolerated for the optimal effect of the drug.

\section{Case 4}

A 60-year-old man with a history of previous myocardial infarction 7 years prior to admission, was admitted to our hospital for general check- 
up. His ECG on admission (Fig. 4, ECG A) disclosed transient complete right bundle branch block with occasional premature ventricular contractions (PVC). Two months after admission, he suddenly developed ventricular fibrillation during a treadmill exercise test. Procainamide, disopyramide, diphenylhydantoin and mexiletine were orally given singly or in combinations without significant effect as shown by arrows indicating ventricular fibrillation. Ventricular fibrillation was induced while the patient was drinking water, taking a bath or was on defecation, not preceded by frequent PVCs or short runs of ventricular tachycardia, but by one PVC superimposed on sinus tachycardia (Fig. 4, ECG B). Left stellectomy was performed, which eliminated PVCs completely, but another attack was induced while he was taking a shower 3 months after surgery. This is a case showing the limits of anti-arrhythmic drug therapy and makes a good indication for implantable automatic defibrillator ${ }^{4}$ when it is available in the future.

\section{DISCUSSION}

In most cases as mentioned above, the efficacy of anti-arrhythmic drugs for an individual patient cannot be predicted from the theoret- ical standpoint, but the treatment must rely on the empirical way for selection of drugs by an experienced physician, depending on the status of the patient. Together with the problem, to what extent surgical intervention can make access to the refractory cases of arrhythmia, medical management of these cases by anti-arrhythmic drugs or others awaits further development in the future.

\section{REFERENCES}

1. MANDEL WJ: Cardiac Arrhy thmias. J B Lippincott Co, Philadelphia, 1980

2. WINKLE RA, GLANTZ SA, HARRISON DC: Pharmacologic therapy of ventricular arrhythmias. Am J Cardiol 36: 629, 1975

3. GREENSPAN AM, HOROWITZ LN, SPIELMAN SR, JOSEPHSON ME: Large dose procainamide therapy for ventricular tachyarrhythmia. $A m J$ Cardiol 46: 453, 1980

4. MIROWSKI M, REID PR, MOWER MM, WATKINS L, GOTT VL, SCHAUBLE JF, LANGER A, HEILMAN MS, KOLENIK SV, FISCHELL RE, WEISFELDT ML: Termination of malignant ventricular arrhythmias with an implanted automatic defibrillator in human beings. $N$ Engl $\mathrm{J} \mathrm{Med}$ 303: 322,1980

5. WINKLE RA, ADELMAN EL, FITZGERALD JW, HARRISON DC: Treatment of recurrent symptomatic ventricular tachycardia. Ann Intern Med 85: 1,1976 